Conocimiento de salud bucal en pacientes mayores de 15 años en Manta, Ecuador

\title{
Conocimiento de salud bucal en pacientes mayores de 15 años en Manta, Ecuador
}

\author{
Oral health knowledge in patients older than 15 years in Manta, Ecuador
}

\section{Conhecimento de saúde oral em pacientes com idade superior a 15 anos em Manta, Equador}

Richard E. Ponce-Andrade ${ }^{\mathrm{I}}$

richardpo-pk@gmail.com

Carlos E. Veliz-Vela II

carlos-veliz123@hotmail.com

Carlos A. Delgado-Delgado III

carlos.delgado55@gmail.com

Recibido: 08 de agosto de 2017 * Corregido: 16 de octubre de 2017 * Aceptado: 25 de noviembre de 2017

I. Diploma Superior en Desarrollo Local y Salud, Diploma Superior en Educación Universitaria Por Competencias, Doctor en Odontología, Docente de la Universidad Laica "Eloy Alfaro" de Manabí, Manta, Manabí, Ecuador.

I. Diploma Superior en Desarrollo Local y Salud, Especialista en Diseño Curricular por Competencias, Doctor en Odontología, Docente de la Universidad Laica "Eloy Alfaro" de Manabí, Manta, Manabí, Ecuador.

III. Diploma Superior en Desarrollo Local y Salud, Diploma Superior en Pedagogía Universitaria, Especialista en Diseño Curricular por Competencias, Doctor en Medicina y Cirugía, Docente de la Universidad Laica "Eloy Alfaro” de Manabí, Manta, Manabí, Ecuador. 


\title{
Resumen
}

Se realizó un estudio descriptivo y transversal para determinar el nivel de conocimiento sobre salud bucal en 200 pacientes mayores de 15 años, que acudieron por primera vez desde enero hasta julio del 2017 a consulta privada de Odontología. Se obtuvo que $87.0 \%$ de los pacientes tenían conocimiento no adecuado sobre las enfermedades bucales más frecuentes y su prevención; $93.5 \%$ sobre la importancia de los dientes, tejidos blandos bucales y auto examen bucal; él 92.0 \% desconocían la importancia de la salud bucal y su repercusión a las enfermedades sistémica y la totalidad sobre los aspectos relacionados con la placa dentobacteriana. Se concluyó que el elevado porcentaje de pacientes con conocimientos no adecuados sobre salud bucal, justificó recomendar la creación de consultas para consejería bucal y desarrollar intervenciones educativas elevar la cultura sanitaria de esa población.

Palabras clave: salud bucal; educación para la salud; prevención.

\begin{abstract}
A descriptive and cross-sectional study was conducted to determine the level of knowledge about oral health in 200 patients over 15 years of age, who attended for the first time from January to July 2017 at the private dentistry office. It was found that $87.0 \%$ of patients had inadequate knowledge about the most frequent oral diseases and their prevention; $93.5 \%$ on the importance of teeth, oral soft tissues and oral self-examination; he $92.0 \%$ were unaware of the importance of oral health and its repercussion to systemic diseases and the totality of the aspects related to dentobacterial plaque. It was concluded that the high percentage of patients with inadequate oral health knowledge justified recommending the creation of consultations for oral counseling and developing educational interventions to raise the health culture of that population.
\end{abstract}

Keywords: oral health; education for health; prevention.

\section{Resumo}

Foi realizado um estudo descritivo e transversal para determinar o nível de conhecimento sobre saúde bucal em 200 pacientes com idade superior a 15 anos, que participaram pela primeira vez de janeiro a julho de 2017 no escritório privado de odontologia. Verificou-se que 87,0\% dos pacientes apresentavam conhecimentos inadequados sobre as doenças bucais mais freqüentes e sua prevenção; $93,5 \%$ sobre a importância dos dentes, tecidos moles orais e auto-exame oral; ele $92,0 \%$ desconheciam 
a importância da saúde bucal e sua repercussão nas doenças sistêmicas e a totalidade dos aspectos relacionados à placa dentobacteriana. Concluiu-se que a alta porcentagem de pacientes com conhecimento inadequado em saúde bucal justificou recomendar a criação de consultas para aconselhamento oral e o desenvolvimento de intervenções educacionais para elevar a cultura da saúde dessa população.

Palavras chave: saúde bucal; educação para a saúde; prevenção.

\section{Introducción}

La boca como instrumento especializado tiene prioridad ontológica sobro todos los demás órganos aparecidos en los seres vivos; es decir, fue el primero en formarse en cualquier tipo de organismo. Antes que el corazón, el cerebro o los pulmones, apareció la boca para que la vida fuera posible, hasta el punto de que ambos fenómenos (boca y vida) pueden considerarse como entidades univocas. ${ }^{1}$

Hay organismos que sobreviven sin aparato locomotor, sin visión e incluso sin sistema nervioso, pero sin ningún tipo de boca para recibir alimentos del exterior la existencia es imposible. ${ }^{1}$

Las enfermedades bucodentales constituyen un importante problema de salud pública por su alta prevalencia y fuerte impacto sobre las personas y la sociedad en términos de dolor, malestar, limitación y discapacidad social y funcional. ${ }^{2}$

Muchos de los factores de riesgo que se asocian a estas enfermedades se relacionan con un inadecuado estilo de vida y unos hábitos insanos adquiridos a lo largo del tiempo. La Educación para la Salud fue definida por la OMS como un campo especial de las Ciencias de la Salud cuyo objetivo es la transmisión de conocimientos y el desarrollo de una conducta encaminada a la conservación de salud del individuo y de la sociedad. La educación es el mecanismo más eficaz del que disponemos para generar un cambio de actitud hacia modos de vida más saludables. ${ }^{3,4,5}$

Las acciones de promoción de salud consisten en comunicar información como una estrategia para lograr cambios en el comportamiento de las personas hacia el cuidado de su salud bucal. Estas acciones no han logrado consolidarse como verdaderos programas de educación en salud. 
Las enfermedades más frecuentes de dientes y encías son la caries y las periodontopatías, las cuales están estrechamente relacionadas con la presencia de placa dentobacteriana producto de una deficiente higiene bucal.

La odontología tiene un futuro promisorio en el camino de la promoción de salud, se trata de crear sanos en la comunidad y no de tratar enfermos en las clínicas, es mucho más fácil educar y dar armas a la población para prevenir y controlar las enfermedades bucales que no brindar servicios terapéuticos.

Bajo estas consideraciones, nos hemos percatado de la falta de conocimientos de la población en los aspectos relacionados con la salud bucal lo cual nos motivó a la realización del estudio motivamos a realizar el estudio.

\section{Material y método}

Se realizó un estudio descriptivo y transversal en 200 pacientes mayores de 15 años que acudieron por primera vez a la consulta odontológica privada correspondiente al periodo enero 2017 a julio 2017, con la finalidad de determinar el nivel de conocimiento que poseían sobre aspectos de la saludbucal.

Se tuvieron en cuenta criterios de inclusión y exclusión previa a la confección de la historia clínica se le informó a cada paciente los objetivos del estudio y la necesidad de su colaboración.

La información se obtuvo a través de una encuesta diseñada al efecto, que contenía 15 preguntas relacionadas con la salud bucal, lo cual sirvió para valorar el grado de conocimiento que poseían en relación con los siguientes temas (cinco de cada tema):

- Conocimiento y control de la placa dentobacteriana

- Enfermedades bucales más frecuentes y su prevención

- Importancia de los dientes, tejidos bucales y auto examen bucal.

- Relación de la salud bucal y las enfermedades generales

Se utilizaron variables epidemiológicas de interés como: edad, sexo y escolaridad

- Edad: Se expresó en grupos de edades:

$-15-18$ 
$-19-34$

$-\quad 35-59$

- 60 y más

- Sexo: Se escogieron sus dos variantes biológicas.

\section{Resultados}

En la tabla 1 se observa que del total de pacientes, 106 correspondió al sexo femenino (53.0 \%) y 94 al masculino (47,0\%). El grupo etáreo más representado fue el de 35-59 años (40,5\%), seguido por el de $19-34$ y 15 a 18, con $33.0 \%$ y 17,5 \%, respectivamente. Solo $9.0 \%$ tenía 60 años y más.

Tabla 1 Pacientes según grupo de edad y sexo

\begin{tabular}{|c|c|c|c|c|c|c|}
\hline \multirow{3}{*}{$\begin{array}{l}\text { Grupo de } \\
\text { edades }\end{array}$} & \multicolumn{4}{|c|}{ Sexo } & \multirow{2}{*}{\multicolumn{2}{|c|}{ Total }} \\
\hline & \multicolumn{2}{|c|}{ Femenino } & \multicolumn{2}{|c|}{ Masculino } & & \\
\hline & No & $\%$ & No & $\%$ & No & $\%$ \\
\hline $15-18$ & 15 & 14.1 & 20 & 21.2 & 35 & 17.5 \\
\hline $19-34$ & 30 & 28.3 & 36 & 38.2 & 66 & 33.0 \\
\hline $35-59$ & 56 & 52.8 & 25 & 26.5 & 81 & 40.5 \\
\hline+60 & 5 & 4.7 & 13 & 13.8 & 18 & 9.0 \\
\hline Total & 106 & 53.0 & 94 & 47.0 & 200 & 100 \\
\hline
\end{tabular}

Se muestra en la tabla 2 el conocimiento que poseían los pacientes sobre las enfermedades bucales más frecuentes y su prevención según grupos de edades, donde se encontró que en 174 de ellos fue no adecuado (87.0 \%) y solamente en 26 (13.0\%) adecuado, el mayor porciento estuvieron ubicados en el grupo de 19 a 34 años (18.1\%). 
Tabla 2. Pacientes según grupos de edades y conocimiento sobre enfermedades bucales más frecuentes y su prevención.

\begin{tabular}{|c|c|c|c|c|}
\hline \multirow{3}{*}{$\begin{array}{l}\text { Grupo de } \\
\text { edades }\end{array}$} & \multicolumn{4}{|c|}{ Nivel de conocimiento } \\
\hline & \multicolumn{2}{|c|}{ Adecuado } & \multicolumn{2}{|c|}{ No adecuado } \\
\hline & No & $\%$ & No & $\%$ \\
\hline $15-18$ & 6 & 17.1 & 29 & 82.8 \\
\hline $19-34$ & 12 & 18.1 & 54 & 81.8 \\
\hline $35-59$ & 8 & 9.8 & 73 & 90.1 \\
\hline+60 & - & - & 18 & 100 \\
\hline Total & 26 & 13.0 & 174 & 87.0 \\
\hline
\end{tabular}

Al analizar el conocimiento que tenían los pacientes sobre importancia de los dientes, tejidos blandos bucales y el auto examen bucal (tabla 4) se encontró que en 187 (93.5 \%) fue no adecuado, entre ellos en todos los mayores de 60 años y en $13(6.5 \%)$ adecuado.

Tabla 3. Pacientes según grupos de edades y conocimientos sobre importancia de los dientes, tejidos blandos bucales y el auto examen bucal.

\begin{tabular}{|l|l|l|l|l|}
\hline \multirow{3}{*}{ edupo de } & \multicolumn{3}{l}{ Nivel de conocimiento } \\
\cline { 2 - 5 } & \multicolumn{2}{|l|}{ Adecuado } & \multicolumn{2}{l|}{ No adecuado } \\
\cline { 2 - 5 } & No & $\%$ & No & $\%$ \\
\hline $15-18$ & 3 & 8.5 & 32 & 91.4 \\
\hline $19-34$ & 4 & 6.0 & 62 & 93.9 \\
\hline $35-59$ & 6 & 7.4 & 75 & 92.5 \\
\hline+60 & - & - & 18 & 100 \\
\hline
\end{tabular}


Conocimiento de salud bucal en pacientes mayores de 15 años en Manta, Ecuador

\begin{tabular}{|l|l|l|l|l|}
\hline Total & 13 & 6.5 & 187 & 93.5 \\
\hline
\end{tabular}

Como se observa en la tabla 4 , el $85.5 \%$ de los pacientes examinados tenían una calidad de higiene bucal mala, predominando en el grupo de 35-59 años (90.1\%). Existió predominio de la presencia de la placa dentobacteriana seguidamente de caries dental

Tabla 4. Pacientes según grupos de edades en relación con la calidad de la higiene bucal.

\begin{tabular}{|l|l|l|l|l|}
\hline \multirow{3}{*}{ Grupo de } & \multicolumn{5}{|c|}{ Nivel de conocimiento } \\
\cline { 2 - 5 } & \multicolumn{3}{|c|}{ Buena } & \multicolumn{2}{c|}{ Mala } \\
\cline { 2 - 5 } & No & $\%$ & No & $\%$ \\
\hline $15-18$ & 10 & 28.5 & 25 & 71.4 \\
\hline $19-34$ & 11 & 16.6 & 55 & 83.3 \\
\hline $35-59$ & 8 & 9.8 & 73 & 90.1 \\
\hline+60 & - & & 18 & 100 \\
\hline Total & 29 & 14.5 & 171 & 85.5 \\
\hline
\end{tabular}

Se indagó lo relacionado a la importancia de la salud bucal y su repercusión a las enfermedades sistémica como la diabetes mellitus, hipertensión arterial, neumonía bacteriana, enfermedades cardiacas el $92.0 \%$ de los pacientes encuestados lo desconocían.

\section{Discusión}

Las enfermedades orales son uno de los problemas principales de salud pública debido a su alta prevalencia, una forma de tratarla a largo plazo es la modificación de conductas de riesgo. Para ello, en una fase inicial debemos establecer los niveles de conocimiento sobre salud oral que tiene la población. ${ }^{6}$ 
Según una investigación sobre consejería de salud bucal para adultos, realizada en un consultorio médico en Cuba, antes de la intervención educativa, 86,7 \% de los pacientes desconocía lo relacionado con la placa dentobacteriana, pero después de esta se logró que 84,4 \% modificara sus conocimientos, lo cual demuestra que el dominio sobre dicho tema es bastante escaso, situación que puede transformarse mediante procedimientos educativos e intervenciones. ${ }^{7}$

García del Prado $^{8}$ y colaboradores en estudios realizados en Venezuela, se encontró que un porcentaje importante de los gerontes requiere de tratamiento protésico rehabilitador y que el nivel de conocimiento de los abuelos sobre salud bucodental fue muy bajo para ambos sexos, de ahí que se haga necesario la elaboración de una estrategia interventiva para mejorar el estado de salud bucal de la población geriátrica venezolana y su nivel de conocimientos sobre salud bucal. Coincidiendo con los resultados de esta investigación con respecto al nulo conocimiento de este grupo poblacional sobre la salud bucal.

La salud de su boca forma parte de un gran todo: su bienestar general. Los dientes y las encías poco saludables a menudo se encuentran en combinación con enfermedades cardíacas y otras enfermedades que pueden poner en riesgo la vida, así como también muchos problemas de salud también pueden afectar su salud bucal. ${ }^{9}$

Las investigaciones cada vez proporcionan más evidencias sobre las conexiones que existen entre la boca y algunas de las enfermedades sistémicas más mortales y costosas. ${ }^{9}$

La identificación temprana de los síntomas o enfermedades bucales puede contribuir al pronto diagnóstico y tratamiento de otras enfermedades sistémicas. Y es que, la salud bucal significa más que dientes saludables. La palabra "bucal" se refiere a la boca, que incluye no sólo los dientes y las encías sino también el paladar, la garganta, la lengua, los labios, las glándulas salivales, los músculos...9

Consideraciones generales de la metodología de trabajo: las encuestas de salud poblacional son herramientas indispensables para identificar y satisfacer las necesidades de salud en su expresión colectiva. Proporcionan datos e información basados en la evidencia para los tomadores de decisiones. ${ }^{10}$ 
Teniendo en cuenta lo antes expuesto se concluye que en el estudio predominó el sexo femenino, con mayor representación en los grupos de edades de 35 a 59 años, el nivel de conocimiento sobre enfermedades bucales más frecuentes y su prevención fue inadecuado al igual que el conocimientos sobre importancia de los dientes, tejidos blandos bucales y el auto examen bucal y su repercusión a las enfermedades sistémicas, fueron evaluados de inadecuados. Al examinar la calidad de la higiene bucal, se valoró de mala, por lo cual recomendamos implementar consultas de consejería bucal y realizar intervenciones a los grupos de edades de la población incluidos en la investigación, que les permita elevar la cultura sanitaria.

\section{Referencias Bibliográficas}

1- Martín Heredia J. Nivel de conocimiento sobre salud buco dental, en la población de Barrio Adentro Peribeca. Municipio Independencia. San Cristóbal. Estado Táchira. 2006 [citado 4 junio 2017]. Disponible en: http:/www.ilustrados.com/tema/10589/Nivel-conocimiento-sobre-saludbucal-poblacion.html

2- Gestal JJ, Smyth E, Cuenca E, Montes A. Epidemiología y prevención de las enfermedades de la cavidad oral: caries dental, periodontopatías y cáncer. En: Piédrola Gil. Medicina Preventiva y Salud Pública. Elsevier Masson: Barcelona; 2008.

3- Bosch Robaina R., Rubio Alonso M., García Hoyos F. Conocimientos sobre salud bucodental y evaluación de higiene oral antes y después de una intervención educativa en niños de 9-10 años. Av Odontoestomatol. 2012 [citado 4 junio 2017]; 28(1): 17-23. Disponible en: http://scielo.isciii.es/scielo.php?script=sci_arttext\&pid=S0213-12852012000100003\&lng=es

4 Salleras L, Fuentes M, Prat Marín A, Garrido P. Educación sanitaria. Conceptos y métodos. En: Piédrola Gil. Medicina Preventiva y Salud Pública. Elsevier Masson: Barcelona; 2008.

5- Rioboo García R. Educación para la Salud Bucodental. Avances. Odontología Preventiva y Odontología Comunitaria. Madrid, 2002: 1321-41.

6- Cueto Urbina, A; Soto Pavez, C, Muñoz Avila, D. Conocimiento, actitudes y prácticas sobre salud oral en niños de 12 años. Rev Chil Salud Pública 2010; Vol 14 (2-3): 363-371 
7- Guibert Reyes W. Procesos psicosociales en la educación en salud y en la prevención del cáncer. Rev Cubana Med Gen Integr 2004; 16 (2): 186-93.

8- García del Prado L, Gutiérrez Hernández G., Quintana Castillo C M, Gutiérrez Hernández M, Fajardo Puig. Caracterización del estado de salud bucal y nivel de conocimientos en pacientes geriátricos Caricuao: Venezuela. Rev haban cienc méd. 2009 [citado 4 junio 2017]; 8(4). Disponible en: http://scielo.sld.cu/scielo.php?script=sci_arttext\&pid=S1729-519X2009000400020\&lng=es.

9- Relación entre la salud dental y las enfermedades sistémicas. 2017. [citado 4 junio 2017]. Disponible en. https://www.clinicadentalurumea.com/salud-dental-y-enfermedades-sistemicas/

10- Andrade FR de, Narvai PC. Inquéritos populacionais como instrumentos de gestão e os modelos de atenção à saúde. Rev. Saúde Pública. 2013; 47 (Suppl 3): 154-160. 